\title{
Literatur - grundsätzlich mehrsprachig!? Das politische Potenzial literarischer Mehrsprachigkeit heute, am Beispiel von Barbi Marković' Superheldinnen
}

\author{
SANDRA VLASTA
}

\begin{abstract}
Literature - Multilingual on Principle?! The Political Potential of Literary Multilingualism Today, using the Example of Barbi Marković's Superheldinnen. Research on literary multilingualism is increasingly based on the assumption that literature per se is multilingual. This is true for concepts such as Mikhail Bakhtin's 'polyphony', in which multilingualism occurs in the form of social, regional and historical variants within one major language. Similarly, it applies to Rainier Grutman's concept of hétérolinguisme, which expands Bakhtin's notion and includes actual language changes. Recently, Till Dembeck has even called for a philology of multilingualism that would accommodate literary multilingualism in literary criticism. Using Barbi Marković's novel Superheldinnen (2016) as an example, I discuss this recent development in multilingual literary studies and analyse concepts, forms and function of literary multilingualism. In so doing, I underline the transcending character of literary multilingualism that expresses itself on various levels: linguistically, formally, medially and with respect to culture. Thus, I aim to illustrate the enormous political potential of literary multilingualism. In fact, multilingualism in literature, as opposed to literature in times of a "monolingual paradigm" (Yasemin Yildiz), poses a political challenge on various levels. Concepts, such as national literature, literary field, but also literary studies and their institutions (i.e. language departments) reach their limits if literature is understood as being multilingual. In the second part of this article, I discuss the difficulties that come with literary prizes, literary studies and the access to the literary field. These often express themselves as concrete problems for individuals who, for instance, have difficulties accessing the literary field.
\end{abstract}

Keywords: multilingualism in literature; political potential; Barbi Marković; Superheldinnen; literary field; literary prizes 
VLASTA

\section{Einleitung $^{1}$}

In der literaturwissenschaftlichen Mehrsprachigkeitsforschung setzt sich immer mehr die Ansicht durch, dass Literatur an sich mehrsprachig sei, egal ob diese Mehrsprachigkeit sich innerhalb einer Sprache findet, wie in Michail Bachtins (1985) Konzept der Polyphonie und dem Konzept der Anderssprachigkeit von Literatur mit Bezug auf Viktor Šklovskij (1973; vgl. Arndt/Naguschewski/Stockhammer 2007) ${ }^{2}$ oder ob sie (auch) traditionelle Sprachgrenzen überschreitet, wie zum Beispiel in Rainier Grutmans (1997) Modell des hétérolinguisme. Till Dembeck (2017b) hat sogar eine Philologie der Mehrsprachigkeit eingefordert, die der Mehrsprachigkeit der Literatur(en) auch auf literaturwissenschaftlicher Ebene Rechnung trägt. Solche Ansätze von literarischer Mehrsprachigkeit haben nicht zuletzt großes politisches Potenzial bzw. sie stellen uns auf verschiedenen Ebenen vor politische Herausforderungen, die sich zu Zeiten eines "monolingual paradigm“ (Yildiz 2012) nicht ergeben. Wenn wir Literatur prinzipiell mehrsprachig denken, stoßen Konzepte wie Nationalliteratur, literarisches Feld, literaturwissenschaftliche Forschung und deren Institutionalisierung in Nationalbzw. zumindest einsprachigen Philologien schnell an ihre Grenzen. Im Gegensatz dazu zeichnen sich mehrsprachige Texte oft durch Prozesse von Grenzüberschreitung aus: in sprachlicher, medialer, formaler sowie kultureller Hinsicht. Einem mehrsprachigen Konzept von Literatur wohnt damit großes politisches Potenzial inne, das Herausforderungen und Probleme mit sich bringt, die ich im Folgenden ausloten möchte. Als Grundlage dafür diskutiere ich im ersten Teil Begriffe und Konzepte sowie Formen und Funktionen literarischer Mehrsprachigkeit anhand eines Textbeispiels Barbi Marković' Roman Superheldinnen (2016). Schließlich thematisiere ich konkrete Herausforderungen literarischer Mehrsprachigkeit und bespreche anhand von Literaturpreisen, dem Zugang zum literarischen Feld und der Literaturwissenschaft beispielhaft die Grenzen der (einsprachig ausgerichteten) Institutionen, wobei ich gleichzeitig das politische Potenzial und die Möglichkeiten zur Veränderung mitdenke.

Teile dieses Artikels stellen eine stark überarbeitete Fassung von Vlasta (2020) dar.

2 Arndt/Naguschewski/Stockhammer verwenden dafür den Begriff der Exophonie (vgl. 2007). 
Literatur - grundsätzlich mehrsprachig!?

\section{Mehrsprachige Autorinnen und Autoren - Barbi Marković}

Literarische Mehrsprachigkeit kann verschiedene Formen und diverse Funktionen haben, wie ich im Folgenden anhand der Autorin Barbi Marković und ihrer Texte zeige. Marković, geboren 1980 in Belgrad, 2006 zum Germanistikstudium nach Wien übersiedelt, wo sie auch heute lebt, schreibt in verschiedenen Sprachen: vor allem auf Serbisch und Deutsch, in ihren Texten finden sich aber auch englische und italienische Elemente und die Figuren in ihrem 2014 uraufgeführten Theaterstück InBetween sprechen Deutsch und Türkisch. Sie kann deshalb mit Steven G. Kellman (2000) als translinguale Autorin bezeichnet werden, d.h. als eine Schriftstellerin, die in einer Sprache, die nicht ihre Erstsprache ist, schreibt (und zudem in mehreren Sprachen). Der Begriff literarische Mehrsprachigkeit kann sich dementsprechend auf die Literatur mehrsprachiger Autorinnen und Autoren beziehen. Beispiele dafür gibt es viele, von Adelbert von Chamisso über Joseph Conrad, Vladimir Nabokov, Elias Canetti und Joseph Brodsky bis zu zeitgenössischen Autorinnen und Autoren wie dem japanisch-britischen Nobelpreisträger Kazuo Ishiguro, der deutschtürkischen Autorin Emine Sevgi Özdamar oder der somalisch-italienischen Schriftstellerin Igiaba Scego.

„Mühsam, aber möglich und auch schön“, so beschreibt Barbi Marković (2020: 195) das Schreiben von Literatur in anderen Sprachen. Trotz der Mühen hat sich die Autorin mit ihren Texten von Beginn an über Sprachgrenzen hinweg bewegt, erstmals mit ihrer serbischen Adaption von Thomas Bernhards Gehen (1971), die 2006 unter dem Titel Izlaženje [Ausgehen] erschienen ist, und, neben zahlreichen kleineren Publikationen, zuletzt in ihrem 2016 auf Deutsch erschienenen Roman Superheldinnen. Izlaženje - eine von der Erzählinstanz selbst als Remix bezeichnete Version des ursprünglichen Texts von Bernhard, den Marković in die Belgrader Clubbing Szene übertragen hat und dessen Protagonistinnen junge Frauen (statt älterer Herren) sind - fand schnell den Weg in die deutschsprachige Literatur: 2009 wurde die deutsche Übersetzung von Mascha Dabić unter dem Titel Ausgehen beim renommierten Suhrkamp Verlag veröffentlicht, dem Verlag, bei dem auch Thomas Bernhards Werke erscheinen. Marković hat für diese Adaption bzw. deren Übersetzung große Aufmerksamkeit von der Literaturkritik erhalten.

Der zuletzt von Barbi Marković publizierte Roman Superheldinnen ist auf Deutsch erschienen, und zwar beim österreichischen Residenz Verlag. Das Buch handelt von drei jungen Frauen - Mascha, Direktorka und die IchErzählerin -, die bei samstäglichen Arbeitstreffen im Café Sette Fontane in Wien, „der Stadt unserer Wahl“ (7), ihre Superkräfte koordinieren und deren Wirkung auf folgende Weise vorausplanen: In einer gemeinsam verfassten wöchentlichen Kolumne in der Wochenzeitschrift Astroblick bitten sie ihre 
VLASTA

Leserinnen und Leser um die zeitgleiche (z.B. am Mittwoch um 18 Uhr) Konzentration auf eine Person in schwierigen Lebensumständen, um diese aber eigentlich mit den auf diese Weise verstärkten „dark[en]“ Kräften des „Blitz[es] des Schicksals“ und der „Auslöschung“ (21) aus dem Leben zu holen - und zwar komplett: Von den Personen bleibt keine Spur mehr, niemand kann sich an sie erinnern. Der Text erzählt von einem dieser Treffen, das gleichzeitig das letzte darstellt, bevor die drei bei einem Casino-Besuch, wiederum dank des Einsatzes ihrer Superkräfte, viel Geld gewinnen und damit endlich auch finanziell den ersehnten Aufstieg in die bürgerliche Mittelschicht schaffen.

Mit diesem Buch, das neben Wien auch in Belgrad, Berlin und Sarajevo angesiedelt ist, hat sich die junge Autorin einerseits ein weiteres Mal in die deutschsprachige Literatur eingeschrieben. Andererseits ist der Roman teilweise auf Deutsch und teilweise auf Serbisch entstanden (die Übersetzung der serbischen Teile hat wiederum Mascha Dabić übernommen), und der Text kann damit als Fortsetzung von Marković' mehrsprachigem Schreiben gesehen werden.

\section{Barbi Marković' Roman Superheldinnen als mehrsprachiger Text}

Marković Roman Superheldinnen zeichnet sich auf mehreren Ebenen durch Mehrsprachigkeit aus: Neben der Autorin sind auch die Figuren einschließlich der Ich-Erzählerin sowie der weiteren, nicht näher spezifizierten Erzählinstanz, die sich in einigen Kapiteln findet, mehrsprachig, außerdem gibt es auf der Ebene des Textes Beispiele für manifeste sowie für latente Mehrsprachigkeit, was nicht zuletzt mit der Technik der Montage zusammenhängt, mit der Marković arbeitet. Ich übernehme die Unterscheidung zwischen manifester und latenter Mehrsprachigkeit von Giulia Radaelli (2011 und 2014), die damit tatsächlich auf der Textoberfläche wahrnehmbare Mehrsprachigkeit (manifest) von nur angedeuteter (latent) differenziert. Mit Bezug auf Radaelli definiert Dembeck manifeste Mehrsprachigkeit als eine Form der Mehrsprachigkeit, die als tatsächlicher Sprachwechsel zwischen „einander wechselseitig intransparenten Idiomen“ (2017a: 150) im Text sichtbar wird. Die manifeste Mehrsprachigkeit entspricht damit dem linguistischen Begriff des code-switching. Bei der latenten Mehrsprachigkeit wird hingegen z.B. durch Hinweise wie ,sagte er auf Englisch ' deutlich, dass in der Figurenrede gerade in einer anderen Sprache gesprochen wird als jener, in der das Gespräch im Text wiedergegeben wird. Auch z.B. Textstellen, die im Rahmen der Diegese offensichtlich in anderen Sprachen verfasst sind (z.B. ein fremdsprachiger Brief, diverse Aufschriften, die einem auf Reisen begegnen), aber in der Hauptsprache des Textes wiedergegeben werden, sind Beispiele für latente Mehrsprachigkeit. 
Ferner ist der Roman Superheldinnen, ebenfalls auf verschiedenen Ebenen, von Übersetzungsvorgängen gekennzeichnet, wie ich im Weiteren ausführe.

Die drei Hauptfiguren Mascha, Direktorka und die Ich-Erzählerin sind mehrsprachig, was zu Beginn des Romans nicht zuletzt durch ihre Herkunft deutlich gemacht wird: „An sich war unsere Geschichte klassisch. Wir drei waren aus den Hauptstädten ärmerer benachbarter Länder hierhergezogen [d.i. nach Wien] und hielten uns nach Kräften über Wasser [...]“ (34). Deutsch sprechen zumindest zwei der drei mit Akzent: „Direktorka und ich waren mit einem bereits fertig ausgebildeten Sprechapparat nach Österreich gekommen, und so stand unser ausländischer Akzent einem normalen Leben im Wege, denn fast jede zufällige Bekanntschaft führte unweigerlich zu einem Gespräch über unsere Herkunft.“ (34) Während Mascha trotz ihres ,Migrationshintergrundes‘ ein „normales Leben“ führen kann, ist das Sprechen mit Akzent für Direktorka und die Ich-Erzählerin eine tägliche Hürde: Immer wieder werden sie auf ihre Herkunft reduziert und Gespräche konzentrieren sich auf diesen Aspekt. Es liegt nahe, dass ihre Anderssprachigkeit mit ein Grund für ihr ökonomisch prekäres Leben ist - wegen ihrer Anderssprachigkeit stoßen die Protagonistinnen an eine ausschließende gesellschaftliche Grenze. Die Mehrsprachigkeit der Protagonistinnen gibt auf diese Weise Anlass zur Reflektion über Sprache, Mehrsprachigkeit, Sprechen und Leben - sie stellt damit eine Form der literarischen Mehrsprachigkeit dar, die ich als Metamehrsprachigkeit bezeichnen möchte (vgl. Vlasta 2019a).

Aus diesen metamehrsprachigen Hinweisen ergeben sich Verweise auf verschiedene Konzepte von Ein- und Mehrsprachigkeit. So wird Mehrsprachigkeit im Roman außerhalb der samstäglichen Arbeitstreffen, die im Mittelpunkt der Handlung stehen, im Sinne des „monolingual paradigm“ dargestellt, wie Yasemin Yildiz (2012) jene Einsprachigkeitsnorm bezeichnet hat, die aus ihrer Sicht ab dem 18. Jahrhundert im Rahmen der Ausbildung nationaler Sprachen, Kulturen und Nationalstaaten propagiert wurde. Die Idee, dass man sich nur in seiner Erstsprache, der ,Muttersprache', gut, korrekt und tatsächlich ausdrücken könne, verweist jedes Sprechen in Fremdsprachen auf eine Position mit geringerer Kompetenz. ,Muttersprache‘ wird damit zu einem Konzept, das im Rahmen des monolingual paradigm eine nicht zuletzt ausschließende Wirkung entfaltet.

Selbst wenn die beiden Protagonistinnen „fertig ausgebildet“ (eine Aussage, die sich schlussendlich nicht nur auf den Sprechapparat bezieht) nach Österreich kommen, so stehen die Wahrnehmung des Akzents und die Reaktionen darauf jeder Anerkennung dieser Ausbildung im Wege. Diese Aussage kann implizit auch als Kommentar über die Autorin und ihr Schreiben bzw. deren Möglichkeiten und Rezeption im deutschsprachigen Feld gelesen 
VLASTA

werden, denn schließlich steht das monolingual paradigm auch für eine Vorstellung von Literatur in der ,Muttersprache', für die Idee, dass man nur in einer, der ,eigenen Sprache' schreiben und auch nur in diese eine Sprache übersetzen könne. Nicht zuletzt ist Steven G. Kellmans Idee einer „translingual imagination“ (2000) als Gegenreaktion auf solche Vorstellungen zu verstehen, genauso wie das aktuelle generelle literaturwissenschaftliche Interesse an literarischer Mehrsprachigkeit. Letzteres wird jedoch gleichzeitig auf gespenstische Weise, wie es David Gramling bezeichnet, von einem „einsprachigen Über-Ich“ begleitet, das immer noch als Norm fungiert, wenngleich es „den alltäglichen Erfahrungen, Repertoires, Referenzen und Praxen der mehrsprachigen Welt kaum Rechnung trägt“ (2017: 37). Allerdings, wie David Martyn festhält, „mehren sich [inzwischen] die Zeichen einer perspektivischen Umkehrung: Nicht die mehrsprachige Literatur ist das Sonderphänomen, sondern die einsprachige." (2014: 40) 3 Wie viele andere Autorinnen und Autoren schreibt Barbi Marković mit ihren Texten gegen Vorstellungen von literarischer Einsprachigkeit an und legt dabei gleichzeitig offen, welche Hindernisse und Hürden Mehrsprachigkeit (in der Literatur, aber auch in der Lebenswelt z.B. in Form eines Akzents) mit sich bringt.

Neben den mehrsprachigen Figuren finden sich auf der Textoberfläche Formen manifester Mehrsprachigkeit. Im Falle von Superheldinnen ist der Großteil des Textes auf Deutsch wiedergegeben, Abweichungen davon in Form von Sprachwechsel und Sprachmischung sind ein Fall von manifester Mehrsprachigkeit. Diese tritt bei Marković vermischt mit Formen von Mehrsprachigkeit auf, die Rainier Grutman (1997) als hétérolinguisme bezeichnet hat: Dieses Konzept erweitert ein traditionelleres Verständnis von Zwei- oder Mehrsprachigkeit um Michail Bachtins Konzept der Polyphonie und bietet damit eine umfassendere Sicht von literarischer Mehrsprachigkeit, zu der auch soziale, regionale und historische Sprachvarianten, also Dialekte, Soziolekte und nichtstandardsprachliche Varianten gezählt werden. Literatur wird in diesem Sinne grundsätzlich als mehrsprachig verstanden.

Bei Marković sind zahlreiche Beispiele für Sprachwechsel und Sprachmischung zu finden, vor allem in den sogenannten "Städte-Texten“ (Preis 2016: 24), wie die Autorin diese Abschnitte selbst bezeichnet. Für diese StädteAbschriften hat die Autorin Beschriftungen gesammelt, die im Stadtraum sichtbar sind: Werbeaufschriften, Lokal- und Firmennamen, Verbote, Gebote, Ankündigungen, etc.: Da reihen sich Werbesprüche an private Mitteilungen, Liebesbotschaften an öffentliche Bekanntmachungen - eine Form der Montage, mit der Marković „Städte oder dieses Stadtgefühl allgemein“ abbilden

3 Vgl. dazu auch Gramling 2016. 
und „das Gefühl der Stadt ins Buch“ (Preis 2016: 24) übertragen möchte. ${ }^{4}$ In Superheldinnen finden sich drei Abschnitte, die auf diese Weise den drei Städten Wien, Sarajevo und Belgrad gewidmet sind. ${ }^{5}$ Außerdem gibt es im Teil 2, in dem die Ich-Erzählerin über ihre Zeit in Berlin (bzw. vor allem am Alexanderplatz) berichtet, montierte Textteile, die ebenfalls die Form von Städte-Texten haben. In diesen Abschnitten werden die Städte-Texte zum Teil mehrsprachig (im Sinne von Grutmans hétérolinguisme) wiedergegeben. So werden Aufschriften und Werbungen in Wien im Dialekt zitiert, wie „Host an Tschick?“6 $(12,15)$, das sich durch den Wien-Stadt-Text zieht. In Berlin wird die Speisekarte eines Fastfood-Restaurants wiedergegeben: „Whopper 3,69 € Double Whopper 4,69€ Big King 3,69€ Big King XXL 4,69 €X-tra Long Chilli Cheese 4,25€ [...]" (74). In Sarajevo ist es einerseits ebenfalls die Werbung, die anderssprachig ist - „Coca-Cola, refresh yourself! Coca-Cola, refresh yourself! Coca-Cola, refresh yourself! Coca-Cola, refresh yourself! Coca-Cola, refresh yourself! Coca-Cola, refresh yourself! Coca-Cola, refresh yourself!“ (108) andererseits sind es Namen von Schriftstellern, die im „Volksbefreiungskrieg“ (111), d.h. im Zweiten Weltkrieg, gefallen sind: „Kalmi Baruh, Zija Dizdarević, Ilija Grbić, Hasan Kikić, Jovan Kršić, Safet Krupić, Džemo Krvavac, Velimir Kovačević, Veselni Masleša, Vasilije Medan, Ognjen Priča, Huso Salčić, Marcel Šnajder, Branko Zagorac." (111-112) Im Fall von Sarajevo und Belgrad werden viele der Beschriftungen übersetzt und auf Deutsch wiedergegeben (d.h. es handelt sich mit Radaelli gesprochen um latente Mehrsprachigkeit), so z.B. „Du weißt, ich weiß, in Wirklichkeit weiß niemand!“ (150) oder „Lade deinen Freund ins Team ein und gewinn mit ihm gemeinsam einen Preis." (151) in Belgrad. Die Städte sind also einerseits von Mehrsprachigkeit gekennzeichnet, was auch an die LeserInnen weitergegeben wird. Andererseits werden die Städte mehrmals übersetzt: Vom öffentlichen Raum in den literarischen Text sowie ins Deutsche. Marković gelingt damit einerseits die beabsichtigte Übertragung des „Gefühl[s] der Stadt“ (Preis 2016: 24) in den Text, andererseits nähert sie die Städte einander sprachlich an und bringt sie den deutschsprachigen Leserinnen und Lesern näher. In diesen Stadtbildern werden außerdem zeithistorische Ereignisse verhandelt, vor allem in den Abschnitten zu Sarajevo

4 In einem Interview gibt Barbi Marković an, dass sie Teile des Grazer Stadt-SchriftProjekts, für das sie während ihres Aufenthalts als Grazer Stadtschreiberin 2011/2012 insgesamt sechs Städte „abgeschrieben“ hat, für den Roman Superheldinnen verarbeitet hat (vgl. Preis 2016: 24).

Vgl. die Kapitel „\#Wien“ (12-15), „\#Sarajevo“ (107-114) und „\#Belgrad“ (148-155).

6 „Hast du eine Zigarette?“ - Die Aufschrift findet sich auf Mülleimern in der Stadt und soll Raucherinnen und Raucher daran erinnern, ihre Zigarettenstummel dort zu entsorgen, anstatt sie auf den Boden zu werfen. 
VLASTA

und Belgrad, in denen Bezüge zu den Jugoslawienkriegen hergestellt werden und deren Hineinwirken in die Gegenwart thematisiert wird. So repräsentieren die oben zitierten Namen der gefallenen Schriftsteller nicht zuletzt die vergangene kulturelle und ethnische Vielfalt Jugoslawiens. In Belgrad hört man Stimmen aus der Kanalisation, die den Menschen zurufen „Mobilisierung für alle Serben!“ (150). Diese Formen von latenter Mehrsprachigkeit, in der andere Sprachen nicht an der Textoberfläche sichtbar werden, sondern nur implizit wahrzunehmen sind, sichern in Marković' Roman nicht zuletzt die Rezeption der eingearbeiteten zeitgeschichtlichen Bezüge. ${ }^{7}$

Allerdings ist die Unterscheidung zwischen manifester und latenter Mehrsprachigkeit in den Texten von Barbi Marković bisweilen schwierig und kann in die Irre führen. Denn Deutsch ist zwar die in Superheldinnen auf der Textoberfläche vor allem sichtbare Sprache, jedoch basiert dieses Original teilweise auf einer Übersetzung, wie Marković in einem Interview erzählt:

Ich habe mit dem ersten Teil auf Deutsch begonnen, wurde dann aber unsicher. Also habe ich den bestehenden Text auf Serbisch übersetzt und neu überarbeitet. Im Prozess bin ich aber draufgekommen, dass ich das Buch doch auf Deutsch brauche, weil ich hier lebe. Die Übersetzerin hat also begonnen, während ich noch weitergeschrieben habe. (Preis 2016: 24)

Das Deutsche stellt damit nur bedingt die Grundsprache des Romans dar, viel eher basiert das Buch auf mehreren Übersetzungsprozessen (die nicht zuletzt dem Zielpublikum geschuldet sind bzw. dem literarischen Feld, in dem sich Marković weiter platzieren möchte oder muss). Diana Hitzke hat gezeigt, dass sich Schreiben und Übersetzung in Marković' erstem Werk Izlaženje vermischen: Übersetzung wird „on its way to writing“ (2016: 442) dargestellt, die Übersetzerin (Hitzke meint hiermit Marković) wird, entgegen üblicher Konventionen (vgl. Venuti 1995; Schahadat 2016), sichtbar „as a writer who shifts between quoting (in translation), variation and adaptation (as writing)“ (Hitzke 2016: 442). Auch für Superheldinnen kann solch eine Vermischung von Schreiben und Übersetzung festgestellt werden, da die Autorin selbst übersetzt und ihre eigene Übersetzung überarbeitet sowie nach der Übersetzung durch Mascha Dabić „noch intensiv mit der deutschen Fassung“ (Preis 2016: 25) gearbeitet hat. Bemerkenswert und kennzeichnend für Marković' Schreiben

\footnotetext{
Ein weiteres Beispiel für latente Mehrsprachigkeit in Superheldinnen ist die Figurenrede, speziell jene der drei Protagonistinnen: Die direkten Reden von Mascha, Direktorka und der Ich-Erzählerin werden im Roman durchgehend auf Deutsch wiedergegeben, genauso wie die Ich-Erzählung selbst. Siehe dazu auch den folgenden Abschnitt zu den Übersetzungsebenen im Roman.
} 
und Arbeitsweise ist das deutliche Sichtbarmachen der Übersetzerin. Mascha Dabić wird auf dem Titelblatt als Übersetzerin genannt, auch eine der Figuren trägt den Namen Mascha - hier wird augenzwinkernd eine Brücke zwischen der Textebene und der Übersetzung hergestellt -, zudem spricht Marković in Interviews über die Rolle der Übersetzung und der Übersetzerin im Entstehungsprozess ihres Romans, wie die oben angeführten Zitate zeigen. ${ }^{8}$ Dieses Sichtbarmachen hat eine doppelte symbolische Bedeutung, da nicht nur generell der Übersetzer oft unsichtbar ist, sondern insbesondere die Übersetzerin, denn, wie Jacques Derrida (1988: 152; vgl. auch Hitzke 2016: 441) hervorhebt, übernehmen oft Frauen die Aufgabe des Übersetzens. Stärker als konkrete Textteile, die übersetzt wurden, steht bei Marković die Figur der Übersetzerin im Vordergrund bzw. die Figuren, denn schließlich übersetzt auch die Autorin ihren Text - vom Deutschen ins Serbische, vom Serbischen ins Deutsche, vom Stadtbild in den Text und so weiter.

Mit diesem Sichtbarmachen und Offenlegen von Übersetzungsprozessen schreibt sich Marković in eine Form der Literatur ein, für die Übersetzung (und damit eine Arbeit mit, zwischen und über die Sprachen hinweg) eine grundlegende Praxis ist und die Wail S. Hassan als „translational literature“ (Hassan 2006 und 2016) bezeichnet hat. Dieses translationale Schreiben stellt Übersetzung als grundlegende Praxis von Kommunikation und kulturellem Transfer dar ohne selbst Übersetzung im klassischen Sinne zu sein: „Translational literature calls into question the implicitness of translation, by expressing the possibilities and limits, the intentions and the consequences of translation in a diverse spectrum of literary forms", wie es Christine Ivanovic und Barbara Seidl formulieren (2016: 958-959). ${ }^{9}$ Auf diese Weise wird Übersetzung zu einem wesentlichen Verfahren literarischer Mehrsprachigkeit und translationale Literatur zu einer typischen Form mehrsprachigen Schreibens. Uljana Wolf bezeichnet dies als „translationale Poetik“ (2018), die translingual, das heißt zwischen den Sprachen angesiedelt ist. Die daraus hervorgehenden Texte sind durch ihre translationale Natur, durch ihre Bewegung zwischen und durch die Sprachen gleichzeitig prädestiniert für das Zirkulieren im Feld der Weltliteratur, zu der David Damrosch „all literary works that circulate beyond their culture of origin, either in translation or in their original language“ (2003: 4) zählt. Barbi Marković’ Literatur hat auf diese Weise von

8 Mascha Dabić ist Übersetzerin und zugleich selbst Autorin, ihr erster Roman Reibungsverluste, der sich bezeichnenderweise ebenfalls mit Übersetzung bzw. dem Dolmetschen auseinandersetzt und in dessen Zentrum eine Übersetzerin steht, ist 2017 erschienen.

9 Für ein Beispiel für translationale Literatur und ein Projekt des Schreibens durch Übersetzung vgl. Christine Ivanovic' (2018) Analyse von Peter Waterhouses Die, should sea be fallen in (2013/15). 
VLASTA

Beginn an die Grenzen des deutschsprachigen literarischen Feldes überschritten.

\section{Grenzen und Herausforderungen literarischer Mehrsprachigkeit}

Die Grenzen, die mehrsprachige Autorinnen und Autoren wie Barbi Marković’ überschreiten, sind gleichzeitig konkrete Herausforderungen literarischer Mehrsprachigkeit, denn sie sind in Institutionen und Konzepten abgebildet und haben konkrete Auswirkungen auf das mehrsprachige Schreiben. Ich möchte dies nun anhand folgender Aspekte erörtern: Literaturpreise, der Zugang zum literarischen Feld und die Literaturwissenschaft.

Im deutschsprachigen Raum gibt und gab es Literaturpreise, die sich gezielt an nicht-deutschsprachige bzw. zugewanderte Autoren und Autorinnen richten (vgl. dazu auch Sievers/Vlasta 2018a und 2018b). Der bekannteste davon ist sicher der in Deutschland vergebene Adelbert-von-Chamisso-Preis, mit dem „die Robert Bosch Stiftung herausragende auf Deutsch schreibende Autoren, deren Werk von einem Kulturwechsel geprägt ist, [ehrt]. Die Preisträger verbindet zudem ein außergewöhnlicher, die deutsche Literatur bereichernder Umgang mit Sprache“ (Robert Bosch Stiftung o.J.). Dieser Preis wurde 2017 zum letzten Mal vergeben, mit dem Hinweis der Robert Bosch Stiftung, dass der Chamisso-Preis eine große Erfolgsgeschichte sei, die man nun guten Gewissens abschließen könne (vgl. Trojanow und Oliver 2016, die sich in einer Reaktion sehr kritisch zum Ende des Chamisso-Preises äußern). Als eine Art ,Nachfolgerpreis' gibt es seit 2019 den in Dresden vergebenen ChamissoPreis/Hellerau, mit dem „herausragende Beiträge zur Gegenwartsliteratur von Autorinnen und Autoren, die aus ihrer je persönlichen Erfahrung eines Sprach- oder Kulturwechsels heraus neue, eigenständige literarische Antworten auf den Wandel unserer modernen, pluralen und globalisierten Welt zu geben vermögen" (Chamisso-Preis/Hellerau o.J.) ausgezeichnet werden. In Österreich gibt es parallel dazu seit 1997 den Literaturwettbewerb, schreiben zwischen den kulturen' der edition exil (vgl. Böckel 2011; Pellegrino 2018; Schwaiger 2016). Er ist gedacht zur Förderung der Literatur von Autorinnen und Autoren, die aus einer anderen Kultur und Erstsprache kommen und in deutscher Sprache schreiben. Für die Autorinnen und Autoren können diese Preise eine Möglichkeit sein, Sichtbarkeit im Literaturbetrieb zu erhalten und von Verlagen und dem Publikum wahrgenommen zu werden. Gleichzeitig bedeuten diese Preise aber eine gewisse Nischenzuordnung, zum Beispiel zur ,Migrationsliteratur' (vgl. Sievers 2016; Vlasta 2013). Darüber sind viele der Autoren und Autorinnen nicht sehr glücklich, denn sie sehen sich in erster Linie als Schriftsteller und Schriftstellerinnen und wollen ihre Werke als 
Literatur (und nicht als soziologische Texte über Migration) gelesen wissen. Die Schubladisierung als Migrationsautor oder -autorin hingegen stellt für viele eine Trennlinie dar, die sie von der eigentlichen deutschsprachigen Literatur abgrenzt (vgl. Disoski 2010; Schwarz 2008).

Alternativ dazu, und das wäre eine mögliche Umsetzung des politischen Potenzials der Grenzüberschreitung von mehrsprachiger Literatur, lässt sich eine Öffnung von allgemeinen Literaturpreisen denken. ${ }^{10}$ Als gelungenes Beispiel dafür möchte ich den Ingeborg-Bachmann-Preis nennen, bei dem 2017 auch Barbi Marković auf Einladung von Klaus Kastberger teilnahm. In einem breiteren Kontext spielt dieser Preis bzw. Wettbewerb seit mittlerweile gut zwei Jahrzehnten eine wichtige Rolle bei der Einführung bzw. der verstärkten Sichtbarmachung anderssprachiger Autorinnen und Autoren in der deutschsprachigen Literatur. Es gibt einige Beispiele für Schriftstellerinnen und Schriftsteller, deren Erstsprache nicht Deutsch ist bzw. die mehrsprachig sind und die beim Ingeborg-Bachmann-Preis teilgenommen haben: So hat 1991 die deutsch-türkische Autorin Emine Sevgi Özdamar den Preis gewonnen, was nicht zuletzt zu einer verstärkten Wahrnehmung von deutsch-türkischer Literatur durch das Publikum, die Kritik und die Literaturwissenschaft geführt hat (vgl. Sievers/Vlasta 2018a; Vlasta 2019b: 152). 2012 hat die gebürtige Russin Olga Martynowa den Preis erhalten, 2013 die Ukrainerin Katja Petrowskaja. 2013 nahm außerdem der gebürtige Brasilianer Zé do Rock am Wettlesen in Klagenfurt teil. Im Jahr 2016 traten neben dem israelischen Autor Tomer Gardi, der wie Marković auf Einladung des Grazer Germanisten Klaus Kastberger dabei war, Marko Dinić (serbisch-österreichischer Autor) und Selim Özdogan (gebürtiger Türke) an. Die Gewinnerin des Ingeborg-BachmannPreises 2016 war mit Sharon Dodua Otoo aus Großbritannien ebenfalls eine Autorin, deren Erstsprache nicht Deutsch, sondern Englisch ist. Auch 2018 ging der Preis an eine Autorin mit anderer Erstsprache, an die gebürtige Ukrainerin Tanja Maljartschuk, die bislang nur auf Ukrainisch publizierte. Der Preis hat somit für allgemeine Bekanntheit und Sichtbarkeit der einzelnen Autorinnen und Autoren gesorgt, aber auch für Aufmerksamkeit für anderssprachige Schriftstellerinnen und Schriftsteller generell im deutschsprachigen literarischen Feld; auf diese Weise wird hier das monolingual paradigm in der deutschsprachigen Literatur aufgelöst.

10 Unter allgemeinen Literaturpreisen verstehe ich hier solche, die, anders als der Chamisso-Preis oder jener der edition exil, nicht aufgrund biographischer Voraussetzungen vergeben werden, sondern auf Basis literarischer Texte oder des Schaffens einer Autorin oder eines Autors in seiner Gesamtheit. 
VLASTA

Ähnlich haben in den letzten Jahren auch der Deutsche sowie der Schweizer Buchpreis zu einer entsprechenden Öffnung des deutschsprachigen Literaturbetriebs beigetragen: Immer wieder wurden Werke mehrsprachiger Autorinnen und Autoren, die auf Deutsch schreiben oder, neben anderen Sprachen, auch auf Deutsch schreiben mit diesen Preisen ausgezeichnet, wie z.B. kürzlich Saša Stanišić (2019), aber auch Terezia Mora (2013) und Melinda Nadj Abonji, die im Jahr 2010 sogar sowohl den Deutschen als auch den Schweizer Buchpreis erhielt.

Diese Öffnung, die zum Beispiel durch Literaturpreise bewirkt werden kann, ist wichtig, denn der Zugang zum literarischen Feld ist für anderssprachige Autorinnen und Autoren zum Teil äußerst schwierig, wie sie selbst erzählen (vgl. den Interviewband Sievers/Englerth/Schwaiger 2017). So sagt zum Beispiel Tomer Gardi in einem aktuellen Interview, dass viele deutsche Verlage seinen in einer experimentellen, von Berliner Jugendslang und dem Deutsch der Einwanderer geprägten Sprache geschriebenen Roman broken german (2016) nicht drucken wollten. Erst der kleine Grazer Verlag Droschl, spezialisiert auf anspruchsvolle, ästhetisch innovative Literatur und situiert an der Peripherie des deutschsprachigen Raums, sei dazu bereit gewesen, so Gardi (vgl. Gardi/Vlasta 2020: 218). Auch die Diskussion, die seinem Auftritt beim Ingeborg-Bachmann-Preis folgte, kreiste unter anderem um die Frage, ob die Bedingung für die Teilnahme an einem Literaturwettbewerb nicht die Sprachkompetenz sein müsste und ob Gardi, der das titelgebende broken German nicht nur schreibt, sondern auch spricht, damit überhaupt teilnahmeberechtigt sei (vgl. Vlasta 2019b und Zusammenfassung Jurydiskussion Tomer Gardi 2016). Der als Kind von Russland nach Österreich eingewanderte Autor Vladimir Vertlib schreibt in einem Essay davon, wie seine ersten Texte von einer Lektorin sprachlich kritisiert wurden: „Regina [d.i. die Lektorin] bemängelte nicht nur den Inhalt meiner Geschichte, sondern vor allem die zahlreichen ,sprachlichen Freiheiten', die ich mir bezüglich Rechtschreibung, Grammatik, Interpunktion und Idiomatik erlaubt hatte" (Vertlib 2012: 30). Sharon Dodua Otoo schließlich hat in ihrer Eröffnungsrede des Ingeborg-Bachmann-Wettbewerbs 2020 einen respektvollen und zugleich freieren Umgang mit „unserer gemeinsamen deutschen Sprachen“ eingefordert, der „souveräne Aushandlungsprozesse" (Otoo 2020: 13) zulasse. Ihr Vorschlag gründet auf persönlichen Erlebnissen: „Leider wird [...] in den überwiegend weißen deutschsprachigen Redaktionen - progressiv wie konservativ - noch immer zu eng am Duden festgehalten. Viel zu oft habe ich die ärgerliche Erfahrung gemacht, dass ein Text von mir im Lektorat,korrigiert' und veröffentlicht wurde, obwohl ich mit meiner gewählten Schreibweise etwas ganz anderes hatte ausdrücken wollen." (Otoo 2020: 13f.) 
Diese Aussagen zeigen, dass der deutschsprachige Literaturbetrieb durchaus noch vom monolingual paradigm geprägt ist und die Vorstellung einer deutschsprachigen Literatur als einer von deutsch(erst)sprachigen Autorinnen und Autorinnen geschriebenen erst noch zu überwinden ist. Mehrsprachigkeit und Anderssprachigkeit führen in diesen Fällen zu Ausgrenzung bzw. lassen die betroffenen Autorinnen und Autoren die Grenzen des Literaturbetriebs deutlich spüren. Dass das nicht immer so war und zudem in verschiedenen kulturellen und sprachlichen Kontexten unterschiedlich ausgeprägt ist, wurde in jüngsten Untersuchungen gezeigt. So hat das Forschungsprojekt Literature on the Move (http://www.litmove.oeaw.ac.at) für den österreichischen Kontext gezeigt, dass anderssprachige Autoren wie Milo Dor oder György Sebestyén bis in die 1950er Jahre Zugang zum literarischen Feld hatten, ohne dass sie eine Ausgrenzung aufgrund ihrer Erstsprache erfahren hätten (vgl. Sievers 2016). In Schweden hingegen wird Literatur in der jeweiligen Muttersprache der MigrantInnen staatlich gefördert, was ein größeres Interesse auch von Seiten der Verlage schafft (Gröndahl/Rantonen 2018: 24).

Die Grenzen betreffen aber auch uns als Literaturwissenschaftlerinnen und Literaturwissenschaftler und die Institutionen, in denen wir tätig sind. Diese Institutionen sind nicht zuletzt in der nach Yildiz ideologisch einsprachig geprägten Periode im späten 18. und 19. Jahrhundert entstanden bzw. dienten dazu, die Idee einer Nationalliteratur, geschrieben in jeweils einer bestimmten Sprache, zu institutionalisieren und zu verfestigen. Germanistik, Anglistik, Italianistik, Polonistik etc. sind jeweils einzelnen Sprachen und den Literaturen in diesen Sprachen gewidmet und gehen damit auch von einer solchen prinzipiellen Einsprachigkeit der Literaturen aus. Das führt zu blinden Flecken, wie zum Beispiel im Fall von Barbi Marković. So wurde ihre erste Erzählung Izlaženje (Ausgehen) - nicht zuletzt aufgrund der Vorlage des mittlerweile kanonischen Autors Thomas Bernhard - zwar vereinzelt von der Literaturwissenschaft rezipiert (und dabei auf die deutsche Übersetzung zurückgegriffen), doch dabei wurden vor allem die formalen Methoden von Marković' Bernhard-Adaption untersucht (Müller-Funk 2010; Simonek 2015). Erst im Rahmen einer stärker für Fragen der Übersetzung und Mehrsprachigkeit sensibilisierten und komparatistisch arbeitenden Literaturwissenschaft hat Diana Hitzke (2016: 426-427; vgl. auch Hitzke 2019) konsequent die drei Texte - Bernhards Gehen, Marković' Izlaženje und dessen deutsche Übersetzung Ausgehen - parallel gelesen und damit jenes Feld von Adaption, Übersetzung und Zirkulation aufgezeigt, das sich zwischen den drei Texten eröffnet. In diesem Feld werden Fragen zum Verhältnis von Original und Übersetzung sowie zu den Ähnlichkeiten und Unterschieden 
VLASTA

zwischen Schreiben, Übersetzen und Adaption aufgeworfen, wie Hitzke in ihrem Beitrag analysiert.

Diese Fragen sind aus meiner Sicht nicht nur in Izlaženje zentral, sondern bilden generell einen Schwerpunkt im mehrsprachigen, transkulturellen Schreiben wie dem von Marković. Eine Vorgangsweise wie die von Hitzke gewählte ist ein Beispiel für eine Philologie der Mehrsprachigkeit, wie Till Dembeck (2017b) sie vorgeschlagen hat. Eine solche Philologie geht von der prinzipiellen Mehrsprachigkeit von Literatur aus, stellt das monolingual paradigm also quasi auf den Kopf. Literaturwissenschaftlerinnen und Literaturwissenschaftler stoßen hier allerdings auf Grenzen - nicht zuletzt auf solche der eigenen sprachlichen Kompetenz. Letzterem kann aber bis zu einem gewissen Grad entgegengewirkt werden, z.B. in Form von Kollaborationen von Literaturwissenschaftlerinnen und Literaturwissenschaftlern mit verschiedenen Sprachkompetenzen.

Schwieriger erscheinen mir die institutionellen Grenzen, in deren Rahmen derzeit Forschung zur literarischen Mehrsprachigkeit nur bedingt gefördert wird und zum Beispiel Qualifikationsschriften zum Thema zwischen den Fronten stehen. Hier treffen, wie im Literaturbetrieb, die einsprachigen institutionellen Wurzeln auf eine Welt, die von mehrsprachigen Individuen, Erfahrungen und Praxen geprägt ist. Das Erscheinen des Handbuchs zu Literatur und Mehrsprachigkeit (Dembeck/Parr 2017), die Gründung einer wissenschaftlichen Buchreihe zum Thema (ebenfalls herausgegeben von Till Dembeck und Rolf Parr), und die Veranstaltung zahlreicher wissenschaftlicher Konferenzen haben die Sichtbarkeit des Phänomens und des Forschungsgebiets wesentlich erhöht und lassen auf eine stärkere Verankerung in der traditionellen Literaturwissenschaft hoffen, die dadurch womöglich gleichzeitig transformiert wird, wie das in Ansätzen schon im Literaturbetrieb passiert (vgl. Vlasta 2018). Wie genau und zu welchen institutionellen Formen von Literaturwissenschaft dies führen wird, ist zum jetzigen Zeitpunkt noch offen.

\author{
Sandra Vlasta \\ savlasta@uni-mainz.de \\ Johannes Gutenberg University Mainz \\ GERMANY
}


Literatur - grundsätzlich mehrsprachig!?

\section{Literaturverzeichnis}

Arndt, S., Naguschewski, D., Stockhammer, R. 2007. Einleitung. Die Unselbstverständlichkeit der Sprache. - S. Arndt, D. Naguschewski, R. Stockhammer, Hrsg., Exophonie. Anders-Sprachigkeit (in) der Literatur. Berlin: Kadmos, 7-27.

Bachtin, M. 1985. Probleme der Poetik Dostoevskijs. Übersetzt von Adelheid Schramm. Frankfurt am Main: Ullstein.

Bernhard, T. 1971. Gehen. Frankfurt am Main: Suhrkamp.

Böckel, V. 2011. Migration in der österreichischen Literatur: Die TrägerInnen des Preises ,schreiben zwischen den kulturen' 2003-2008. Saarbrücken: VDM Verlag Dr. Müller.

Chamisso-Preis/Hellerau. o.J.. Über den Preis. - https://www.chamissopreishellerau. de (06.08.2021).

Dabić, M. 2017. Reibungsverluste. Wien: Edition Atelier.

Damrosch, D. 2003. What is World Literature? Princeton: Princeton University Press.

Dembeck, T. 2017a. Sprachwechsel/Sprachmischung. - T. Dembeck, R. Parr, Hrsg., Literatur und Mehrsprachigkeit. Ein Handbuch. Tübingen: Narr Francke Attempto, 125-166.

Dembeck, T. 2017b. There Is No Such Thing as a Monolingual Text! New Tools for Literary Scholarship. - Webportal www.polyphonie.at 4 (1/2017).

Dembeck, T., Parr, R., eds. 2017. Literatur und Mehrsprachigkeit. Ein Handbuch. Tübingen: Narr Francke Attempto.

Derrida, J. 1988. Roundtable on Translation. - C. McDonald, Hrsg., The Ear of the Other. Otobiography, Transference, Translation. Texts and Discussions with Jacques Derrida. Lincoln and London: University of Nebraska Press, 93-161.

Disoski, M. 2010. Ich mache Literatur und Punkt! - Der Standard, 15.2.2010. https:// www.derstandard.at/story/1265851881267/interview-ich-mache-literatur-undpunkt (06.08.2021).

Gardi, T., Vlasta, S. 2020. Gespräch mit Tomer Gardi. Im E-Mail-Gespräch mit Tomer Gardi war Sandra Vlasta im Mai 2018. - B. Siller, S. Vlasta, Hrsg., Literarische (Mehr-)Sprachreflexionen. Wien: Praesens, 214-221.

Gramling, D. 2016. The Invention of Monolingualism. New York and London: Bloomsbury Academic Publishing.

Gramling, D. 2017. Einsprachigkeit, Mehrsprachigkeit, Sprachigkeit. - T. Dembeck, R. Parr, Hrsg., Literatur und Mehrsprachigkeit. Ein Handbuch. Tübingen: Narr Francke Attempto, 35-44.

Gröndahl, S., Rantonen, E. 2018. Migrants and literature in Finland and Sweden. Helsinki: Finnish Literature Society. https://doi.org/10.21435/sflit.11

Grutman, R. 1997. Des langues qui résonnent. L’hétérolinguisme au XIXe siècle québécois. Montréal: Fides-CÉTUQ.

Hassan, W. S. 2006. Agency and Translational Literature: Ahdaf Soueif's The Map of Love. - PMLA, 121 (3), 753-768. https://doi.org/10.1632/003081206X142869

Hassan, W. S. 2016. Translational Literature and the Pleasures of Exile. - PMLA, 131 (5), 1435-1443. https://doi.org/10.1632/pmla.2016.131.5.1435 
VLASTA

Hitzke, D. 2016. Translation, Adaptation, Circulation. Barbara Markovićs Izlaženje. Journal of World Literature, 1, 426-444. https://doi.org/10.1163/2405648000103009

Hitzke, D. 2019. Nach der Einsprachigkeit. Slavisch-deutsche Texte transkulturell. Berlin u.a.: Peter Lang. https://doi.org/10.3726/b16372

Ivanovic, C. 2018. ,We are translated men': Translational Literature and Migration. Austrian Studies, 26, 106-123. https://doi.org/10.5699/austrianstudies.26.2018. 0106

Ivanovic, C., Seidl, B. 2016. What is Translational Literature and How to Classify it? Crowd-sourcing as a Starting Point for Corpus Building and Type Distinction in Comparative Literature. - Proceedings of the Fourth International Conference on Technological Ecosystems for enhancing Multiculturality TEEM 2016. New York: ACM Digital Library, 957-963. https://doi.org/10.1145/3012430.3012632

Kellman, S. G. 2000. The Translingual Imagination. Lincoln, Nebraska: University of Nebraska Press.

Literature on the Move (o.J.). http://www.litmove.oeaw.ac.at (06.08.2021).

Marković, B. 2006. Izlaženje. Belgrad: Rende.

Marković, B. 2009. Ausgehen. Übersetzt von Mascha Dabić. Frankfurt am Main: Suhrkamp.

Marković, B. 2014. InBetween. Uraufführung 21.3.2014, Ethos Uluslararası Tiyatro Festivali, Ankara.

Marković, B. 2016. Superheldinnen. Mit Übersetzungen von Mascha Dabić. Salzburg: Residenz.

Marković, B. 2020. Mühsam, aber möglich und auch schön. Über die Textarbeit in mehreren Sprachen. - B. Siller, S. Vlasta, Hrsg., Literarische (Mehr-)Sprachreflexionen. Wien: Praesens, 195-196.

Martyn, D. 2014. Es gab keine Mehrsprachigkeit, bevor es nicht Einsprachigkeit gab: Ansätze zu einer Archäologie der Sprachigkeit. - T. Dembeck, G. Mein, Hrsg., Philologie und Mehrsprachigkeit. Heidelberg: Universitätsverlag Winter, 39-51.

Müller-Funk, W. 2010. Kontext, Intertextualität und Übersetzung. Thesen und Hypothesen samt einer exemplarischen Analyse: Thomas Bernhards „Gehen“, Barbi Marković” „Izlaženje“. - H. van Uffelen, W. Kriegleder, E. Mengel, A. Woldan, Hrsg., Literatur im Kontext: ein gegenseitiges Entbergen. Wien: Praesens, 143162.

Otoo, S. D. 2020. Dürfen Schwarze Blumen malen? Klagenfurter Rede zur Literatur 2020. Klagenfurt/Celovec: Edition Meerauge, Johannes Heyn.

Pellegrino, R. 2018. Der Preis schreiben zwischen den kulturen: Ein Beispiel für Mehrsprachigkeit und Mehrkulturalität in der österreichischen Literaturszene. - M. Bürger-Koftis, R. Pellegrino, S. Vlasta, Hrsg., wokommstduher? Inter-, Multi- und Transkulturalität im österreichischen Kontext. Wien: Praesens, 71-98.

Preis, T. 2016. Meine Spezialität ist die sogenannte Halbironie. Barbi Marković im Gespräch mit dem Anzeiger über Texte, die uns umgeben, warum Tauben die perfekten Comicfiguren sind und ihren neuen Roman Superheldinnen (Residenz). Anzeiger, 4, 24-25. 
Literatur - grundsätzlich mehrsprachig!?

Radaelli, G. 2014. Literarische Mehrsprachigkeit. Ein Beschreibungsmodell (und seine Grenzen) am Beispiel von Peter Waterhouses Das Klangtal. - T. Dembeck, G. Mein, Hrsg., Philologie und Mehrsprachigkeit. Heidelberg: Winter, 156-182.

Radaelli, G. 2011. Literarische Mehrsprachigkeit. Sprachwechsel bei Elias Canetti und Ingeborg Bachmann. Berlin: Akademie Verlag. https://doi.org/10.1524/9783050053592

Robert Bosch Stiftung (o.J.). Adelbert-von-Chamisso-Preis der Robert Bosch Stiftung. https://www.bosch-stiftung.de/de/projekt/adelbert-von-chamisso-preis-der-robert-bosch-stiftung (01.06.2020).

Schahadat, S. 2016. Sichtbare Übersetzer - transkulturelle Biographien. Am Beispiel von Karl Dedecius und Ilma Rakusa. - S. Schahadat, Š. Zbytovský, Hrsg., Übersetzungslandschaften. Themen und Akteure der Literaturübersetzung in Ost- und Mitteleuropa. Bielefeld: Transcript, 19-40. https://doi.org/10.14361/9783839433027-001

Schwaiger, S. 2016. Über die Schwelle. Literatur und Migration um das Kulturzentrum exil. Wien: Praesens.

Schwarz, C. 2008. Ich bin ein begeisterter Deutscher. - Wirtschaftswoche 12.10.2008. http://www.wiwo.de/lifestyle/ich-bin-ein-begeisterter-deutscher-374009/ (06.08.2021).

Sievers, W. 2016. Grenzüberschreitungen: Ein literatursoziologischer Blick auf die lange Geschichte von Literatur und Migration. - W. Sievers, Hrsg., Grenzüberschreitungen: Ein literatursoziologischer Blick auf die lange Geschichte von Literatur und Migration. Wien: Böhlau, 10-38. https://doi.org/10.7767/9783205204664-002

Sievers, W., Englerth, H., Schwaiger, S. 2017. ich zeig dir, wo die krebse überwintern. Wien: edition exil.

Sievers, W., Vlasta, S. 2018a. From the Exclusion of Individual Authors to the Transnationalisation of the Literary Field: Immigrant and Ethnic-Minority Writing in Germany. - W. Sievers, S. Vlasta, Hrsg., Immigrant and Ethnic-Minority Writers since 1945. Fourteen National Contexts in Europe and Beyond. Leiden: Brill/Rodopi, 219-258.

Sievers, W., Vlasta, S. 2018b. New Austria, Old Roots: Writers of Immigrant Origin in Austria. - W. Sievers, S. Vlasta, Hrsg., Immigrant and Ethnic-Minority Writers since 1945. Fourteen National Contexts in Europe and Beyond. Leiden: Brill/Rodopi, 43-76.

Simonek, S. 2015. Remix als Verfahren der Popliteratur (am Beispiel von Thomas Bernhard und Barbi Marković). - J. Doschek, S. Simonek, Hrsg., Slawische Popkultur. Wien: Polnische Akademie der Wissenschaften - Wissenschaftliches Zentrum in Wien, 169-184.

Šklovskij, V. 1972 [1914]. Die Auferweckung des Wortes. - W.-D. Stempel, Hrsg., Texte russischer Formalisten II. München: Fink, 3-17.

Trojanow, I., Oliver, J. F. A. 2016. Ade, Chamisso-Preis? - Frankfurter Allgemeine Zeitung, 21.9.2016. http://www.faz.net/aktuell/feuilleton/debatten/kritik-anbosch-stiftung-ade-chamisso-preis-14443175.html (06.08.2021).

Venuti, L. 1995. The Translator's Invisibility. A History of Translation. London, New York: Routledge. 
VLASTA

Vertlib, V. 2012. Der Autor und sein Alter. - V. Vertlib, Ich und die Eingeborenen. Dresden: Thelem, 29-32.

Vlasta, S. 2013. 'Literature of Migration': A New Trend in 'Austrian Literature'? M. Gratzke, M.-A. Hutton, C. Whitehead, eds., Readings in Twenty-First-Century European Literatures. Oxford u.a.: Peter Lang, 405-430.

Vlasta, S. 2018. Literature, Migration and the Transnationalisation of Literary Fields Austria and Great Britain. - 900 Transnazionale 2. https://ojs.uniroma1.it/index. php/900Transnazionale/article/view/14028 (06.08.2021).

Vlasta, S. 2019a. Literarische Mehrsprachigkeit in österreichischer Migrationsliteratur - Formen, Funktionen und Rezeption bei Tomer Gardi, Semier Insayif und Vladimir Vertlib. - A. Leben, A. Koron, eds., Literarische Mehrsprachigkeit im österreichischen und slowenischen Kontext. Tübingen: Narr Francke Attempto, 245-257.

Vlasta, S. 2019b. ,Was ist ihre Arbeit hier, in Prosa der deutschsprachige Sprach? Mehrsprachige Räume der Begegnung und Empathie in Tomer Gardis Roman broken german. - M. Acker, A. Fleig, M. Lüthjohann, eds., Affektivität und Mehrsprachigkeit. Dynamiken der deutschsprachigen Gegenwartsliteratur. Tübingen: Narr Francke Attempto, 143-157.

Vlasta, S. 2020. Mehrsprachigkeit, Montage, Übersetzung. Barbi Marković' translinguale, transkulturelle und translationale (Welt-)Literatur. - B. Siller, S. Vlasta, eds., Literarische (Mehr-)Sprachreflexionen. Wien: Praesens, 197-213.

Wolf, U. 2018. Wovon wir reden, wenn wir von mehrsprachiger Lyrik reden. https:// stadtsprachen.de/fr/text/wovon-wir-reden-wenn-wir-von-mehrsprachiger-lyrikreden/ (01.06.2020).

Yildiz, Y. 2012. Beyond the Mother Tongue. The Postmonolingual Condition. New York: Fordham University Press. https://doi.org/10.2307/j.ctt13x0cqr

Zusammenfassung Jurydiskussion Tomer Gardi. 2016. Website Ingeborg-BachmannPreis Archiv 2016. http://bachmannpreis.orf.at/stories/2783362/ (06.08.2021). 